\title{
Analysis of Recent Cases of Intestinal Tuberculosis in Japan
}

\author{
Tateki Yamane ${ }^{1}$, Akira Umeda ${ }^{2}$ and Hitoshi Shimao ${ }^{3}$
}

\begin{abstract}
In Japan, which ranks first among developed countries in the incidence of tuberculosis, intestinal tuberculosis should be regarded as an important disease. However, few studies of this condition have recently been reported. We analyzed nine patients treated at our hospital during and after 2000. Our results differ from those of previous studies in that many of the patients were healthy, not elderly, had primary intestinal tuberculosis and presented with non-gastrointestinal symptoms. All seven patients tested were found to be whole blood interferon gamma release assay (IGRA)-positive. These results suggest that IGRA is useful as an adjunct method for diagnosing intestinal tuberculosis.
\end{abstract}

Key words: intestinal tuberculosis, recent analysis, Japanese cases

(Intern Med 53: 957-962, 2014)

(DOI: 10.2169/internalmedicine.53.1862)

\section{Introduction}

The incidence of tuberculosis in Japan was 17.7 per 100,000 population in 2011, which currently ranks first among developed countries and is markedly higher than that observed in the second-ranking country. The year-on-year rate of decrease in the incidence of tuberculosis in Japan was $11 \%$ from the postwar period until the 1970 s, then declined to $3 \%$ in the $1980 \mathrm{~s}$ and transiently increased again in the latter half of the 1990s. Therefore, intestinal tuberculosis remains an important gastrointestinal disease. However, few studies of this condition have been reported in the recent literature. In the present study, we collected and analyzed data from patients examined in our hospital during and after 2000.

\section{Case Report}

Nine patients with active intestinal tuberculosis were treated in our hospital and affiliated institutions between 2000 and 2012. The subjects were analyzed regarding their age, gender, symptoms, clinical characteristics, site of involvement in the gastrointestinal tract, endoscopic findings, histopathological findings of endoscopic biopsy specimens, culture findings, PCR findings and tuberculin skin test (TST) and whole blood interferon gamma release assay (IGRA) results in addition to the presence of extra-intestinal lesions. All patients were cured after receiving antituberculous therapy and therefore definitively diagnosed with intestinal tuberculosis, although the timing of infection was unclear.

The patients included five men and four women, ranging in age from 47 to 80 years, with a mean age of $63.8 \pm 11.8$ years. Five patients presented with gastrointestinal symptoms, including abdominal pain, diarrhea and bloody stools. Of the remaining four patients, two experienced fever and fatigue, while the other two were asymptomatic and positive for fecal occult blood on regular medical check-ups. All patients were HIV-negative. One patient each was diabetic, a heavy drinker and elderly ( 80 years old): the remaining six patients were healthy (Table 1).

The terminal ileum and right colon were involved in seven patients, with the transverse or left colon involved in one case each. The duodenum was also involved in one case. Endoscopy showed annular ulcers and atrophic scarred mucosa, characteristics of intestinal tuberculosis, in six and four patients, respectively (Table 2).

The histopathological examinations of the biopsy specimens showed no caseating granulomas, although non-

\footnotetext{
${ }^{1}$ Division of Gastroenterology, Department of Internal Medicine, The International University of Health and Welfare, Shioya Hospital, Japan, ${ }^{2}$ Division of Pulmonology, Department of Internal Medicine, The International University of Health and Welfare, Shioya Hospital, Japan and ${ }^{3}$ Department of Surgery, The International University of Health and Welfare, Shioya Hospital, Japan Received for publication October 22, 2013; Accepted for publication November 28, 2013 Correspondence to Dr. Tateki Yamane, yamane@iuhw.ac.jp
} 
Table 1. Clinical Profile of Nine Patients

\begin{tabular}{|c|c|c|c|c|}
\hline Case & Age (y.o.) & Sex & Clinical symptom & $\begin{array}{l}\text { Underlying disease- } \\
\text { Patient characteristics }\end{array}$ \\
\hline 1 & 49 & male & diarrhea, abdominal pain & none \\
\hline 2 & 70 & female & fever, general fatigue & $\mathrm{DM}$, angina pectoris \\
\hline 3 & 80 & female & $\begin{array}{l}\text { diarrhea, abdominal pain, } \\
\text { abdominal fullness }\end{array}$ & aged, hypertension \\
\hline 4 & 47 & male & none (stool occult positive) & none \\
\hline 5 & 60 & male & diarrhea, abdominal pain & heavy drinker \\
\hline 6 & 72 & male & abdominal pain & hypertension \\
\hline 7 & 72 & female & none (stool occult positive) & hypertension \\
\hline 8 & 50 & male & $\begin{array}{l}\text { fever, joint pain, general } \\
\text { fatigue }\end{array}$ & none \\
\hline 9 & 74 & female & $\begin{array}{l}\text { diarrhea, bloody stool, } \\
\text { abdominal pain }\end{array}$ & none \\
\hline
\end{tabular}

Table 2. Lesion Site of Digestive Tract and Endoscopic Findings

\begin{tabular}{cll}
\hline Case & \multicolumn{1}{c}{ Lesion site } & \multicolumn{1}{c}{ Endoscopic findings } \\
\hline 1 & ileum end-ascending colon & multiple erosions, mucosal edema \\
2 & ileum end-ascending colon & annular ulcer, multiple erosions, scarring mucosa \\
3 & transverse colon & solitary ulcer \\
4 & ileum end-ascending colon, & annular ulcer, scarring mucosa, \\
& 2nd portion of duodenum & swelling of P. Vater \\
5 & ileum end-ascending colon & annular ulcer, multiple erosions, scarring mucosa \\
6 & ileum end-ascending colon & annular ulcer, multiple erosions, scarring mucosa \\
7 & ileum end-ascending colon & annular ulcer, multiple erosions \\
8 & ileum end-cecum & multiple irregular ulcers-erosions \\
9 & descending colon-sigmoid & annular ulcer, multiple erosions \\
& colon & \\
\hline
\end{tabular}

Table 3. Pathological Findings of Biopsy Specimens

\begin{tabular}{cccc}
\hline Case & $\begin{array}{c}\text { Caseating } \\
\text { granuloma }\end{array}$ & $\begin{array}{c}\text { Non-caseating } \\
\text { granuloma }\end{array}$ & $\begin{array}{c}\text { Ziehl-Neelsen } \\
\text { staining }\end{array}$ \\
\hline 1 & - & + & + \\
2 & - & + & - \\
3 & - & + & + \\
4 & - & + & - \\
5 & - & - & - \\
6 & - & + & - \\
7 & - & - & - \\
8 & - & - & - \\
9 & - & + & - \\
\hline
\end{tabular}

caseating granulomas were observed in six specimens, two of which exhibited positive acid-fast staining: Ziehl-Neelsen (ZN) staining-results (Table 3).

Biopsy and stool specimens were culture- and PCRpositive in one and two patients, respectively. TST was positive in seven patients and negative in two patients. IGRA, performed in seven patients, was positive in all cases. Two patients had extra-intestinal lesions (one case of miliary tuberculosis, one case of tubercular peritonitis): however, no cases of concomitant pulmonary tuberculosis were observed (Table 4).

\section{Case presentation}

Patient 1 was a 49-year-old man who complained of diarrhea, abdominal pain and fever. Colonoscopy demonstrated multiple areas of erosion and edema in the terminal ileum and ascending colon (Fig. 1A). A biopsy showed noncaseating granulomas (Fig. 1B), in which $\mathrm{ZN}$ staining revealed numerous acid-fast bacilli (Fig. 1C). Chest CT disclosed multiple granular shadows in both lungs (Fig. 1D), thus resulting in a diagnosis of miliary tuberculosis.

Patient 3 was an 80-year-old woman who complained of diarrhea with abdominal pain and distention. Colonoscopy showed a solitary ulcer surrounded by multiple small areas of elevation in the mid-transverse colon (Fig. 2A). A biopsy revealed non-caseating granulomas (Fig. 2B), and ZN staining demonstrated acid-fast bacilli (Fig. 2C). Abdominal CT demonstrated ascites (Fig. 2D), and a further evaluation resulted in a diagnosis of tubercular peritonitis.

Patient 4 was a 47-year-old man who was asymptomatic: however, because his stool was found to be positive for occult blood at a regular medical check-up, he underwent colonoscopy, which revealed annular ulcers in the ascending colon and atrophic scarred mucosa in the cecum (Fig. 3A, B). Upper gastrointestinal endoscopy showed a swollen duodenal major papilla (Fig. 3C), a biopsy of which disclosed non-caseating granulomas (Fig. 3D). 
Table 4. Bacteriological Findings, Results of Tuberculin Skin Test (TST) and Whole Blood Interferon Gamma Release Assay (IGRA) and Extraintestinal Lesion

\begin{tabular}{cccccc}
\hline Case & $\begin{array}{c}\text { Biopsyspecimen } \\
\text { culture-PCR }\end{array}$ & $\begin{array}{c}\text { Stool } \\
\text { culture-PCR }\end{array}$ & TST & IGRA & Extra-intestinal lesion \\
\hline 1 & + & + & + & $\begin{array}{c}\text { not } \\
\text { examined }\end{array}$ & miliary tuberculosis \\
2 & - & - & - & not & none \\
3 & - & - & + & + & tubercular peritonitis \\
4 & - & - & + & + & none \\
5 & - & - & + & + & none \\
6 & - & - & + & + & none \\
7 & - & + & - & + & none \\
8 & - & - & + & + & none \\
9 & - & - & + & + & none \\
\hline
\end{tabular}
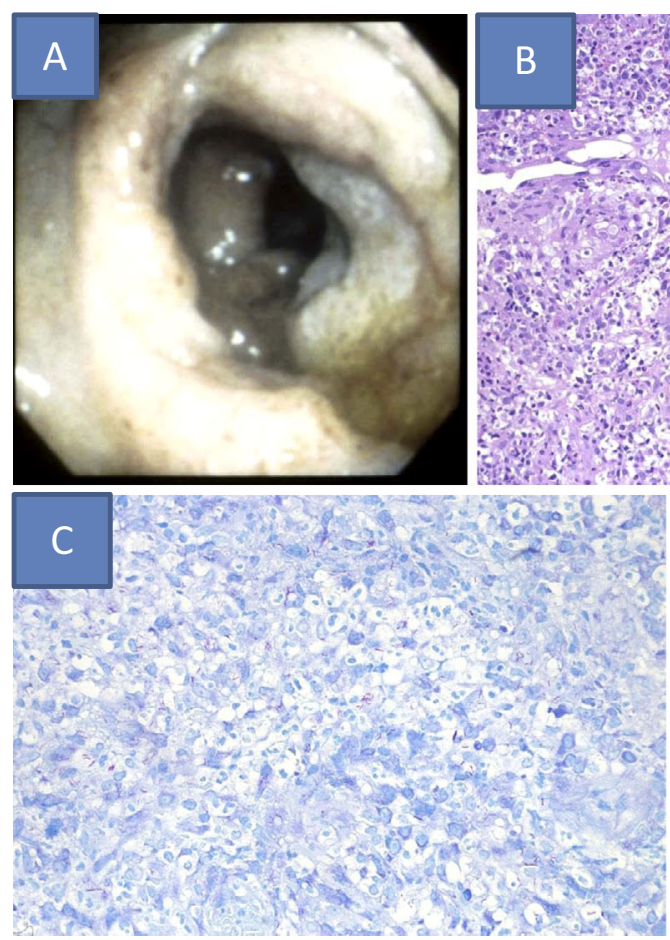

Figure 1. A: Colonoscopy showed multiple erosions and mucosal edema in the right colon. B: Histopathological findings. A non-caseating granuloma was found (arrow) (Hematoxylin and Eosin staining). C: Histopathological findings. Numerous acid-fast bacilli were found (Ziehl-Neelsen staining). D: A chest CT scan showed multiple granular shadows in both lungs.

\section{Discussion}

The incidence of tuberculosis after infection with Mycobacterium tuberculosis is reported to be $30 \%$. It is generally accepted that $50 \%$ of affected patients develop tuberculosis within three months to two years after infection, while the remaining $50 \%$ develop the disease at some point during their lifetime. It is thought that $15-20 \%$ of the Japanese population is already infected. Furthermore, it has been reported that the higher the age, the higher the rate of tubercu- losis infection (with rates of $1 \%, 15 \%$ and $60 \%$ among individuals in their $20 \mathrm{~s}, 50 \mathrm{~s}$ and $70 \mathrm{~s}$, respectively), and the incidence of tuberculosis increases with age. However, in the present study, the mean age of the patients was 63.8 years (no tendency toward an elderly population). In addition, although the timing of infection was unclear, many of the patients were suspected of having developed the disease immediately after infection. Patients with intestinal tuberculosis commonly present with gastrointestinal symptoms, such as abdominal pain, diarrhea and bloody stools. However, nongastrointestinal symptoms were relatively common in this 


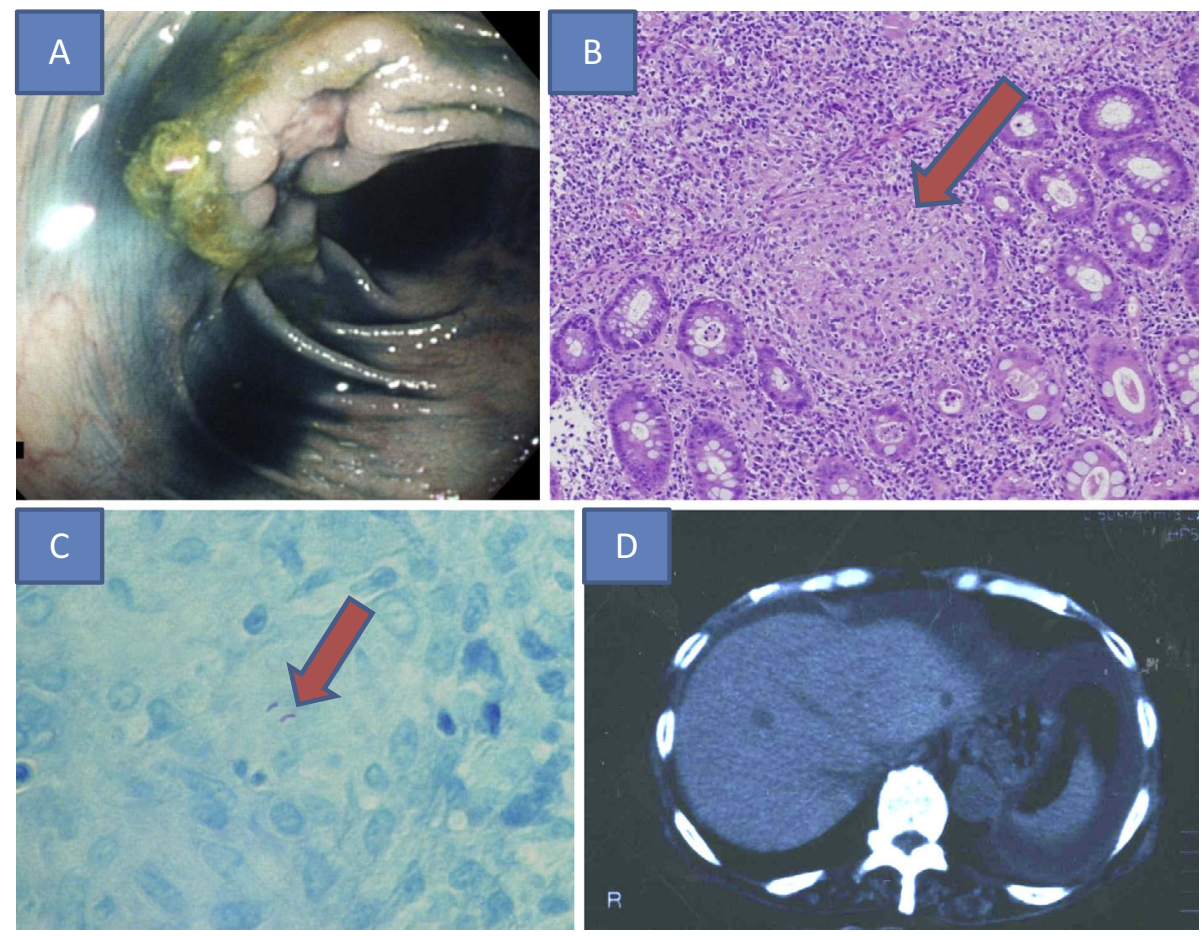

Figure 2. A: Colonoscopy showed a solitary ulcer in the transverse colon (Indigo carmine spraying view). B: Histopathological findings. A non-caseating granuloma was found (arrow) (Hematoxylin and Eosin staining). C: Histopathological findings. Acid-fast bacilli were found (arrow) (Ziehl-Neelsen staining). D: An abdominal CT scan showed ascites.
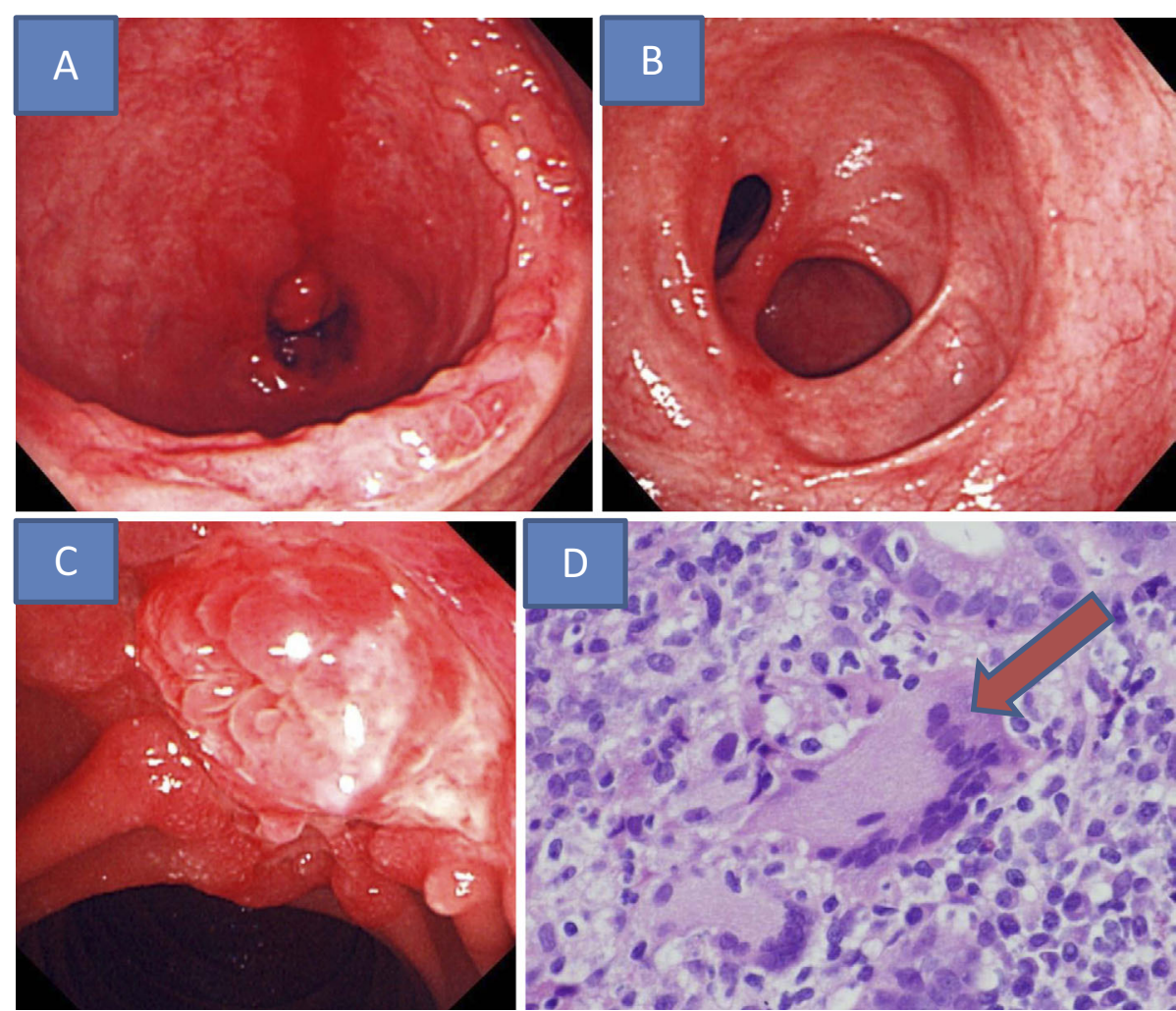

Figure 3. A: Colonoscopy showed annular ulcers in the ascending colon. B: Colonoscopy revealed atrophic scarred mucosa in the cecum. C: Upper gastrointestinal endoscopy showed a swollen duodenal major papilla. D: Histopathological findings of the duodenal biopsy specimen. A non-caseating granuloma was found (arrow) (Hematoxylin and Eosin staining). 
study (four patients, or 44.4\%). In two of the patients with non-gastrointestinal symptoms, abdominal CT performed to evaluate the cause of fever and fatigue showed intestinal wall thickening, resulting in a diagnosis of intestinal tuberculosis. In the remaining two asymptomatic patients, the finding of a positive fecal occult-blood test at a regular medical check-up triggered a diagnosis of the disease. Recently, there has been concern regarding the increase in the incidence of tuberculosis associated with immune dysfunction $(1,2)$. However, in the present study, as many as six patients with intestinal tuberculosis $(66.7 \%)$ were healthy.

Previous studies have reported that intestinal tuberculosis involves the duodenum (15\%), jejunum (15\%), ileum (55\%), ileocecal region $(62.5 \%)$ and colon $(47.5 \%)$ in Japan, in contrast to the ileocecal region $(66.1 \%)$, ascending colon (30.9\%), transverse colon (20.4\%), descending colon (22.8\%), sigmoid colon (11.1\%) and rectum (6\%) in Europe and the United States. The sites of predilection for intestinal tuberculosis include the ileocecal region and its vicinity where lymphoid tissue is abundant, and, in this study, the terminal ileum and right colon were involved in as many as seven patients $(77.8 \%)$.

The imaging findings of intestinal tuberculosis are variable, consisting primarily of tubercular nodules that develop after the initial trapping of bacteria in lymphoid follicles, small ulcers that result from the necrosis of tubercular nodules, large ulcers that form from the fusion of small ulcers, annular ulcers formed along the lymphatic vessels in the transverse plane of the bowel and the presence of atrophic scarred mucosa following the healing of recurrent ulcers $(3,4)$. Among these findings, annular ulceration and atrophic scarred mucosa are characteristic of intestinal tuberculosis $(3,4)$ and have been reported to occur in $69 \%$ and $56 \%$ of patients, respectively (4). Similarly, in this study, these findings were present in six $(66.7 \%)$ and four patients $(44.4 \%)$, respectively.

It is difficult to detect caseating granulomas, a finding that establishes the diagnosis of intestinal tuberculosis, by histopathologically examining endoscopic biopsy specimens (5-11), as such tissues are obtained primarily from submucosa or deeper. No caseating granulomas were detected in this study. On the other hand, it is generally accepted that the rate of detection of non-caseating granulomas, which are suggestive of intestinal tuberculosis, is relatively high. In this study, such lesions were detected in six patients $(66.7 \%)$. The rate of positive acid-fast staining is said to be low (7-10), and specimens from only two patients $(22.2 \%)$ were positive on $\mathrm{ZN}$ staining in this study.

Previous studies have reported rate of culture- and PCRpositive findings for Mycobacterium tuberculosis on biopsy specimens of 6-54.5 (6-9) and 30-64.1\% (7, 9), respectively. In this study, the specimens from only one patient $(11.1 \%)$ were culture- and PCR-positive. The rates culture- and PCRpositive findings for the bacterium in stool specimens are reportedly very low; however, in this study, the specimens from two patients $(22.2 \%)$ were culture- and PCR-positive, thus suggesting the significance of such findings.

IGRA, performed in seven patients, was positive in all cases. It has been reported that this test is not influenced by previous BCG vaccination or non-tuberculous acid-fast bacterial infection, with a sensitivity and specificity for the diagnosis of pulmonary tuberculosis of $89 \%$ and $98 \%$ (12), respectively, making it a promising alternative to TST. The utility of IGRA has been reported in cases of intestinal tuberculosis, especially in the differential diagnosis of Crohn's disease $(13,14)$, and the results of this study suggest its usefulness as an adjunct to the diagnosis of intestinal tuberculosis. However, IGRA has been reported to shows falsenegative results in some cases, particularly those involving patients with hypoalbuminemia or an immunologically compromised status (15-17), and some IGRA-false negative cases of intestinal tuberculosis have been reported $(18,19)$. Therefore, the results of IGRA should be evaluated carefully.

In this study, no patients had concomitant pulmonary tuberculosis. One patient had tubercular peritonitis, which had presumably developed as a result of secondary spread from the intestinal tuberculosis to peritoneal lymph nodes. Therefore, we concluded that eight patients $(88.9 \%)$, excluding one patient with miliary tuberculosis, had primary intestinal tuberculosis. In Japan, recent studies have reported an increase in the incidence of primary intestinal tuberculosis: $50 \%$ in the $80 \mathrm{~s}$ and $66.7 \%$ in the 90 s. Our results suggest a further recent increase in the incidence of this disease.

In conclusion, the results of the present study suggest that affected patients can develop intestinal tuberculosis, even those who are not elderly or immunologically compromised, and that the disease infrequently presents with gastrointestinal symptoms. Furthermore, the incidence of primary intestinal tuberculosis is increasing, and IGRA is useful as a diagnostic adjunct.

\section{The authors state that they have no Conflict of Interest (COI).}

\section{References}

1. Mori T. The transition and problems of tubercular prevail. Nippon Naika Gakkai Zasshi 89: 834-840, 2000 (in Japanese).

2. Hiwatari N, Noguchi M, Suzuki H, et al. Present status in the diagnosis of intestinal tuberculosis. Stomach and Intestine 30: 497506, 1995 (in Japanese).

3. Hirata I. Inflammatory bowel disease and differential disease. Nippon Naika Gakkai Zasshi 98: 44-53, 2009 (in Japanese).

4. Igarashi M, Katsumata T, Naito Y, et al. X-ray and endoscopic diagnosis of colonic tuberculosis. Stomach and Intestine 30: 515524, 1995 (in Japanese).

5. Shan S, Thomas V, Mathan M, et al. Colonoscopic study of 50 patients with colonic tuberculosis. Gut 33: 347-351, 1992.

6. Bhargava DK, Kushwaha AKS, Dasarathy S, et al. Endoscopic diagnosis of segmental colonic tuberculosis. Gastrointest Endosc 38: 571-574, 1992.

7. Kim KM, Lee A, Choi KY, et al. Intestinal tuberculosis: clinicopathologic analysis and diagnosis by endoscopic biopsy. Am J Gastroenterol 93: 606-609, 1998. 
8. Sato S, Yao K, Yao T, et al. Colonoscopy in the diagnosis of intestinal tuberculosis inasymptomatic patients. Gastrointest Endosc 59: 362-368, 2004

9. Gan HT, Chen YQ, Quyang Q, et al. Differentiation between intestinal tuberculosis and Crohn's disease in endoscopic biopsy specimens by polymerase chain reaction. Am J Gastroenterol 97: 1446-1451, 2002.

10. Misra SP, Misra V, Dwivedi M, et al. Colonic tuberculosis: clinical features, endoscopic appearance and management. J Gastoroenterol Hepatol 14: 723-729, 1999.

11. Pulimood AB, Ramakrishana BS, Kurian G, et al. Endoscopic mucosal biopsies are useful in distinguishing granulomatous colitis due to Crohn's disease from tuberculosis. Gut 45: 537-541, 1999.

12. Mori T, Sakatani M, Yamagishi F, et al. Specific detection of tuberculosis infection with an interferon- $\gamma$-based assay using new antigens. Am J Respir Crit Care Med 170: 59-64, 2004.

13. Li Y, Zhang LF, Liu XQ, et al. The role of in vitro interferon $\gamma$ release assay in differentiating intestinal tuberculosis from Crohn's disease in China. J Crohns Colitis 6: 317-323, 2012.

14. Lei Y, Yi FM, Zhao J, et al. Utility of in vitro interferon $\gamma$-release assay in differential diagnosis between intestinal tuberculosis and Crohn's disease. J Dig Dis 14: 68-75, 2013.

15. Kobashi Y, Sugiu T, Shimizu H, et al. Clinical evaluation of the TSPOT. TB test for patients with indeterminate results on the QuantiFERON TB-2G test. Intern Med 48: 137-142, 2009.

16. Bocchino M, Matarese A, Bellofiore B, et al. Performance of two commercial blood IFN- $\gamma$ release assays for the detection of Mycobacterium tuberculosis infection in patient candidates for antiTNF- $\alpha$ treatment. Eur J Clin Microbiol Infect 27: 907-913, 2008.

17. Brock I, Ruhwald M, Lundgren B, Westh $H$, Mathiesen LR, Ravn P. Latent tuberculosis in HIV positive, diagnosed by the M. tuberculosis specific interferon- $\gamma$ test. Respir Res 7: 56, 2006.

18. Inaba N, Chibana $Y$, Saifuku $Y$, et al. Clinical evaluation of the QuantiFERON TB-2G test in two patients with active intestinal tuberculosis. Gastroenterol Endosc 53: 3310-3316, 2011 (in Japanese, Abstract in English).

19. Nakamura M, Omiya N, Honda W, et al. Inflammatory small bowel disease-intestinal tuberculosis. Stomach and Intestine $\mathbf{4 3}$ : 591-596, 2008 (in Japanese).

(C) 2014 The Japanese Society of Internal Medicine http://www.naika.or.jp/imonline/index.html 